\title{
Nutrition-Based Approaches in Clinical Trials Targeting Cognitive Function: Highlights of the CTAD 2020
}

\author{
K.V. Giudici $i^{1}$ \\ 1. Gerontopole of Toulouse, Institute of Aging, Toulouse University Hospital (CHU Toulouse), Toulouse, France.

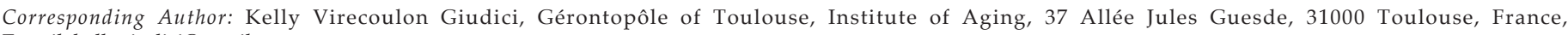 \\ E-mail: kellygiudici@gmail.com \\ J Prev Alz Dis 2021;2(8):118-122 \\ Published online February 7, 2021, http:/ / dx.doi.org/10.14283/jpad.2021.6
}

The Clinical Trials on Alzheimer's Disease (CTAD) 2020 conference was the stage for researchers from all over the world to present their recent and ongoing research focused on potential Alzheimer's disease (AD) treatments and prevention of cognitive decline. Among a varied range of topics, nutritional aspects arose as possibilities of treatments towards the promotion of a healthy aging. Among the discussed themes, supplementation of omega3 polyunsaturated fatty acids and multi-nutrient approaches were presented, suggesting that long-term supplementation (i.e., over 3 years) might be needed for observing positive effects on cognitive performance. Trials testing ketogenic agents and carbohydraterestricted diet were also presented and showed promising effects on improving cognitive function of mildcognitive impaired (MCI) and pre-diabetic individuals, respectively, in a short-term way (i.e. after 3 to 6 months). The combination of some of the nutritional approaches with physical activity interventions raises the question on whether they would individually perform in a similar way. Promising therapies involving nutrition appear to be safe and well tolerated by volunteers. Failures on achieving positive findings raise questions on whether they were driven by specific characteristics of the studied populations, insufficient doses or duration of treatment. Notwithstanding, current evidence on the applicability of nutrition-based approaches as AD treatments are encouraging but demand further research on the topic.

\section{Introduction}

As the population's life expectancy becomes longer and the prevalence of Alzheimer's disease (AD) increases worldwide, measures protecting cognitive function and promoting healthy aging become of extreme relevance to public health. The Clinical Trials on Alzheimer's Disease (CTAD) conference annually welcomes the most recent findings from clinical trials of $\mathrm{AD}$ treatments, bringing light to profitable discussions on their successes and failures, in order to allow future research to be better designed and more effective. Its most recent edition, which occurred virtually due to the COVID-19 pandemic, Received January 27, 2021

Accepted for publication January 29, 2021 welcomed over 1,500 participants from more than 40 different countries from November 4th to 7th, 2020, and provided opportunities for researchers to share what they have learned from their latest and ongoing studies.

Among a wide range of investigations on treatments aiming to slow cognitive decline and fully understand the pathophysiological mechanisms leading to $\mathrm{AD}$, trials targeting nutritional pathways stand out as possibilities of nonpharmacological interventions. The present article discuss the main findings of the studies that have used nutritional approaches, exploring the characteristics that could have possibly contributed to their efficacy or failure.

\section{Single-nutrient approaches}

The effects of omega- 3 polyunsaturated fatty acids (PUFA) on fighting inflammation and oxidative stress are well stablished in literature $(1,2)$. Docosahexaenoic acid (DHA) and eicosapentaenoic acid (EPA), mainly, have been shown to have neuroprotective abilities through both vascular and neuronal mechanisms $(3,4)$, being associated with reduced white matter hyperintensities (WMH) accumulation and delayed neurodegeneration $(5,6)$. In this sense, trials based on omega-3 PUFA supplementation have been developed. The doubleblind, placebo-controlled PUFA Trial (NCT01953705) provided marine omega-3 PUFA to North-American adults free of dementia but with suboptimum plasma omega-3 $(<110 \mu \mathrm{g} / \mathrm{mL})$ and magnetic resonance imaging (MRI)-derived WMH burden $(\geq 5 \mathrm{~cm} 3)$, aged 75 years and older, for 3 years (7). Participants took daily doses of $1,650 \mathrm{mg}$ of omega-3 PUFA (975mg of EPA and $675 \mathrm{mg}$ of DHA) or placebo. Data presented in CTAD 2020 showed that WMH progression was slowed over 3 years among participants that adhered to study protocol, while no effects were observed among the modified intention to treat (mITT) cohort presenting at least one followup MRI. No differences were seen in medial temporal lobe, total brain or ventricular volume changes, nor in executive function Z-score though. The given dose and duration appeared to be safe, since no differences in adverse events between active group and placebo were 
observed (8).

Another study testing the effects of omega-3 PUFA supplementation on cognitive function among community-dwelling older adults is the multicenter, randomized, double-blind controlled Multidomain Alzheimer Preventive Trial (MAPT) (NCT01513252), performed in France and Monaco with subjects aged 70 years or more reporting subjective memory complaints. MAPT also provided omega-3 PUFA supplementation over 3 years (in a daily dose of $225 \mathrm{mg}$ of EPA and $800 \mathrm{mg}$ of DHA, combined or not to a multidomain intervention based on nutritional counseling, exercise advice and cognitive training), however was unable to observe a protective role of interventions on cognitive function among its mITT population (9). The MAPT team then wondered if specific subgroups would respond differently to treatment. Indeed, participants of MAPT Study who were amyloid- $\beta(\mathrm{A} \beta)$ positive (as measured by positron emission tomography - PET scan) have been shown to respond to the multidomain intervention (combined or not with omega-3 supplementation) (10). Defining amyloid status by PET is, however, less accessible and more expensive. Blood-based biomarkers, instead, have shown high reliability to predict neurodegeneration, as $A \beta$ peptides - which are highly associated with PET amyloid status and $A \beta$ in cerebrospinal fluid (CSF) - in a cost-effective and less invasive way (11-13). On the recent study presented at CTAD 2020 conference, participants had their plasma $A \beta 42$ and $A \beta 40$ variants assessed by a high-precision immunoprecipitation and liquid chromatography-mass spectrometry assay in a subsample of participants of MAPT Study, at the end of the first year of follow-up. While no effects of MAPT interventions were observed for cognitive function among the intention-to-treat (ITT) $A \beta$ negative group $(n=322)$ nor in the group with low $A \beta 42 / 40$ ratio (i.e. $A \beta$ positive, $n=161$ ), the perprotocol group of $A \beta$ positive participants $(n=154)$ presented lower cognitive decline after receiving the omega-3 supplementation combined with the multidomain intervention. The omega-3 supplementation alone, however, did not present effects on cognitive performance among the per-protocol $\mathrm{A} \beta$ positive group. In addition, the observed benefits did not persist after 2 years post-intervention (14).

Findings from the PUFA Trial and the MAPT Study point towards the utility of classifying individuals based on their omega-3 status and brain characteristics (such as WMH and amyloid burden) to optimize the early identification of at-risk subjects to whom future interventions would be more profitable. For amyloid status specifically, the assessment of blood $\mathrm{A} \beta$ peptides emerge as a promising method for screening nondemented populations with memory complaints. The daily dose of omega-3 PUFA should also be importantly considered. Based on these studies, it is possible that a higher dose (as the one provided to participants of the
PUFA Trial) might be needed for achieving positive responses after 3 years. Moreover, findings suggest that omega-3 treatments may lose their effects upon discontinuity.

\section{Multi-nutrient approaches}

In addition to trials focusing only on omega-3 PUFA properties, two other trials presented at CTAD 2020 included nutritional approaches based on multi-nutrient supplementation. Such choice was stimulated by considering the multifactorial mechanisms leading to cognitive decline, and to previous investigations showing promising effects of the combination of nutrients in cognitive outcomes among animals $(15,16)$ and humans (17-19).

The LipiDiDiet Trial (Dutch Trial Register number NTR1705) is a double-blind, multi-center randomized controlled trial performed in Finland, Germany, Netherlands and Sweden with 311 older adults presenting prodromal AD (defined according to the International Working Group - IWG-1 criteria). Following an initial 24-month intervention composed of daily receiving a $125 \mathrm{~mL}$ drink containing a multi-nutrient combination (named Fortasyn Connect) or an isocaloric placebo drink, participants could opt to continue in the trial for a maximum of 72 months of intervention, and another 24 months of observational follow-up. Previous publications reporting findings after 24 months of followup have shown that supplementation with the multinutrient drink (containing EPA, DHA, phospholipids, choline, uridine monophosphate, vitamins B6, B9, B12, $\mathrm{C}, \mathrm{E}$ and selenium) had no significant effect on cognitive function measured by a composite neuropsychological test battery (NTB) (20). In CTAD 2020, researchers reported their findings over a total of 36 months of intervention following the initial randomization. After 3 years, significantly lower cognitive decline and brain atrophy were observed for those taking the multinutrient drink. According to the NTB and the clinically relevant measure of Clinical Dementia Rating - Sum of Boxes (CDR-SB), the active group declined respectively $45 \%$ and $60 \%$ less compared to the placebo group. In addition to these clinically detectable benefits, the multinutrient drink presented a good safety profile and high compliance throughout this study $(21,22)$.

The NOLAN Study (NCT03080675) has also tested the effectiveness of a multi-nutrient oral supplement on cognitive function and brain volume of communitydwelling older adults, as also on erythrocyte omega-3 and plasma homocysteine (biomarkers with a capacity to underlie an attenuation in cognitive decline during aging (23-25)). The nutritional blend was composed by two soft capsules and a powdered drink mix to be solved in cold water, containing omega-3 PUFA (DHA and EPA), choline, vitamins B1, B2, B3, B5, B6, B7, B9, B12, E, C, D, selenium and citrulline, and offered daily for one year to 
participants presenting subjective memory complaints. In the meanwhile, placebo identical doses were offered to the control group, composed of volunteers expressing the same concerns. After this period, the nutritional blend successfully increased erythrocyte omega-3 and reduced plasma homocysteine concentrations, while both biomarkers worsened in the other group. Results, however, did not support its use for preventing cognitive decline over a 1-year range. Marginal significance was observed for hippocampal atrophy in the left hemisphere (with the active group presenting a lower volume decrease) (26), raising the question if a longer follow-up would have brought positive effects on brain measures.

Indeed, the limited duration of the follow-up in the NOLAN study is believed to have prevented the identification of positive findings, what is reinforced by results of the LipiDiDiet Trial showing that 36 months, but not 24, were able to deliver positive effects of the multi-nutrient intervention on cognitive performance. Taken together, the presented results suggest that benefits may get apparent or more pronounced with long-term use, highlighting the need for longer trials. The dose of each nutrient may also have contributed to the lack of effects over 1 or 2 years.

\section{Other dietary approaches}

Another nutritional approach used in a trial presented at CTAD 2020 was the oral supplementation with ketone bodies. Brain function is mainly fueled by glucose. In the insufficiency of circulating glucose (which occurs after the depletion of glycogen stores caused by a long fasting state), ketones (acetoacetate and beta-hydroxybutyrate) are naturally produced to keep brain functioning (27). In mild cognitive impairment (MCI) and $\mathrm{AD}$, brain glucose uptake is reduced $(28,29)$. In contrast, uptake of ketones has been shown to remain normal in both MCI and mild-moderate $\operatorname{AD}(28,30,31)$. Previous research have suggested that endogenous or exogenous sources of ketones may improve energy metabolism in both MCI and $\mathrm{AD}$ subjects (32). To test if such improvement might also benefit cognitive performance, the randomized, placebo-controlled Benefic Trial (NCT02551419) supplemented MCI subjects with an oral nutritional supplement composed of ketogenic medium chain triglycerides (kMCT), and compared them to controls taking placebo for 6 months. The nutritional supplement was a lactose-free skim milk emulsion containing $15 \mathrm{~g}$ of kMCT to be taken twice a day. Similarly, an energyequivalent placebo providing $13 \mathrm{~g}$ of non-ketogenic vegetable oil was offered to the control group twice a day. Brain ketones, glucose PET and plasma ketone response were assessed in subgroups. After 6 months, brain ketone significantly increased in the intervention group, while no changes in plasma ketone response were observed for both groups. At the end of follow-up, global brain ketone uptake doubled and cognitive performance (as measured by tests comprising episodic memory, executive function and language) improved in the active group only, in a probable clinically meaningful way. However, this group also presented increased interleukine- 8 , but not other inflammatory markers. Overall, the consistent plasma ketone response suggested no metabolic adaptation to the oral kMCT drink after 6 months of treatment, with safety and good acceptability from participants $(33,34)$.

Also using a nutrition-based approach, the Blood Flow Improvement Trial (BFIT) (NCT03117829) was designed to test whether an intervention based on exercise and carbohydrate-restricted diet (CRD) would reduce insulin resistance (IR) and improve cognitive function. The potential relationship between IR and cognitive performance stands on evidence showing that type 2 diabetes is associated with increased risk of cognitive impairment (35) and of MCI conversion to dementia $(36,37)$, what is believed to occur through vascular damage and dysfunctions in glucose, insulin and amyloid metabolism (38). Such evidence raises the question if reversing IR in midlife would benefit cognition. To test this hypothesis, the BFIT Study was a stepped wedge cluster randomized trial that performed a 12-week intervention composed of exercise and CRD among 29 pre-diabetic, cognitively unimpaired participants (mean age $57.9 \pm 5.1$ years), followed by a 6-month post-intervention phase. The exercise arm of treatment consisted of 50 minutes of supervised moderate intensity aerobic exercise three times per week, unsupervised exercise twice a week, and weekly classes of behavioral change to promote program adherence. The CRD was instructed by a dietitian and contained less than $100 \mathrm{~g}$ of carbohydrates per day. Participants were told to avoid or restrict grains, sugars, legumes, starchy vegetables and fruits, but not carbohydrate from dairy, condiments, nuts and seeds, and were instructed to further keep the regimen for six months more. Exercise and CRD successfully improved markers of glucose metabolism, executive function and verbal memory, even after 6 months post-intervention (39). However, the lack of a control group in this study should be noted as a limitation. Ongoing analyses with data of this trial will investigate the impact of intervention on cerebral blood flow, and test whether improvements in cerebral blood flow and cardiorespiratory fitness would mediate enhancements in cognitive performance.

The Benefic Trial presenting a ketogenic approach with $\mathrm{MCI}$ volunteers suggests this to be a potential treatment pathway to reduce the risk of $\mathrm{MCI}$ progressing towards AD. In a similar way, findings from the BFIT Study based on physical activity and carbohydrate restriction approach with pre-diabetic subjects raise the possibility that preventing pre-diabetes progression towards diabetes would simultaneously prevent cognitive impairment. Future investigations with different populations are required to test whether the CRD without the exercise arm would mimic the benefits, and to help 
establishing these nutrition-based possibilities as part of the new generation of $\mathrm{AD}$ treatments.

\section{Take home lessons}

- Single-nutrient approaches based on omega-3 PUFA supplementation suggested that long-term supply (i.e., more than 3 years) might be needed for observing positive effects on cognitive performance. Findings from the PUFA Trial and the MAPT Study also raise the possibility that treatments may differ according to omega-3 status and brain characteristics (such as $\mathrm{WMH}$ and amyloid burden) of individuals.

- Multi-nutrient approaches comprising omega-3 PUFA, choline, minerals and vitamins are believed to promote synergistic actions that increase effect magnitude on protecting cognitive function. At CTAD 2020, trials testing this novel approach provided mixed evidence, suggesting that medium to long-term follow-ups might be needed in order to identify positive effects. In the LipiDiDiet Trial, reduced progression of hippocampal, ventricular and whole brain atrophy were observed after 36 months, but not after 2 years of intervention. The NOLAN Study was unable to find positive effects in cognitive function over a 1-year frame, but successfully increased erythrocyte omega-3 and decreased plasma homocysteine.

- Supplements driving ketogenic status in the Benefic Trial were shown to directly enhance brain energy status by the increased supply of ketones, resulting in improvements in cognitive performance of older adults with MCI after 6 months, and to be safe. The ketogenic approach for protecting cognitive function deserves further investigations.

- Among people with impaired glucose regulation, a combined 12-week intervention of carbohydraterestricted diet and exercise was shown to reduce insulin resistance and improve cognitive function, even after 6 months post-intervention. Whether the dietary arm of the BFIT Study would provide similar benefits without the increased physical activity demands additional exploring.

- Nutrition-based treatments were offered as oral supplements. Products were well tolerated and no indication for safety concerns was observed. None of the provided nutrients achieved their tolerable upper intake level (UL). On the other hand, the lack of success in some studies comes up with the possibility that the provided dose of nutrients may have been insufficient.

In spite of the unquestionable high value of drug treatments, nutritional approaches stand out as alternatives to prevent and/or slow cognitive decline, contributing to define the next generation of $\mathrm{AD}$ preventive measures and treatments, in order to promote a healthier aging process. Optimizing treatments through nutrition might be especially interesting in the sense that, differently from drugs with specific targets, nutrients are widely recognized by different types of cells and are part of several metabolic mechanisms. Considering that older people are at higher risk of malnutrition (40) and micronutrients deficiency $(41,42)$, interventions based on nutrients may also help treating and/or preventing other comorbidities typically developed with the advancing of age. The exact way on how the aforementioned interventions or similar ones may do that deserves further exploring.

By joining worldwide leaders in the treatment of AD together, the CTAD conference allowed profitable discussions on candidate therapeutics and methodological issues related to this field of research, bringing crucial elements for optimizing the development of effective $\mathrm{AD}$ treatments. The next CTAD conference, which will be held in Boston (USA) from November 9th through 12th, 2021, is expected to bring additional and novel elements that will help designing successful clinical trials and increasing diversity of future AD treatments.

Abbreviations: A $\beta$ : amyloid- $\beta$; AD: Alzheimer's disease; BFIT: Blood Flow Improvement Trial; CDR-SB: Clinical Dementia Rating - Sum of Boxes; CRD: carbohydrate-restricted diet; CSF: cerebrospinal fluid; CTAD: Clinical Trials on Alzheimer's Disease; DHA: docosahexaenoic acid; EPA: eicosapentaenoic acid; IR: insulin resistance; ITT: intention to treat; IWG: International Working Group; kMCT: ketogenic medium chain triglycerides; MAPT: Multidomain Alzheimer Preventive Trial; MCI: mild cognitive impairment; mITT: modified intention to treat; MRI: magnetic resonance imaging; PET: positron emission tomography; PUFA: polyunsaturated fatty acids; UL: tolerable upper intake level; WMH: white matter hyperintensities.

\section{Conflicts of interest: None.}

Open Access: This article is distributed under the terms of the Creative Commons Attribution 4.0 International License (http:/ / creativecommons.org/ licenses/by/4.0/), which permits use, duplication, adaptation, distribution and reproduction in any medium or format, as long as you give appropriate credit to the original author(s) and the source, provide a link to the Creative Commons license and indicate if changes were made.

\section{References}

1. Giordano E, Visioli F. Long-chain omega 3 fatty acids: molecular bases of potential antioxidant actions. Prostaglandins Leukot Essent Fatty Acids. 2014;90(1):1-4.

2. Shibabaw T. Omega-3 polyunsaturated fatty acids: anti-inflammatory and anti-hypertriglyceridemia mechanisms in cardiovascular disease. Mol Cell Biochem. 2020 Nov 11; doi: 10.1007/s11010-020-03965-7. Online ahead of print.

3. Dyall SC. Long-chain omega-3 fatty acids and the brain: a review of the independent and shared effects of EPA, DPA and DHA. Front Aging Neurosci. 2015;7:52

4. Chitre NM, Moniri NH, Murnane KS. Omega-3 Fatty Acids as Druggable Therapeutics for Neurodegenerative Disorders. CNS Neurol Disord Drug Targets. 2019;18(10):735-49.

5. Bowman GL, Silbert LC, Howieson D, et al. Nutrient biomarker patterns, cognitive function, and MRI measures of brain aging. Neurology. 2012;78(4):241-9.

6. Tan ZS, Harris WS, Beiser AS, et al. Red blood cell $\omega-3$ fatty acid levels and markers of accelerated brain aging. Neurology. 2012;78(9):658-64.

7. Bowman GL, Silbert LC, Dodge HH, et al. Randomized Trial of Marine n-3 Polyunsaturated Fatty Acids for the Prevention of Cerebral Small Vessel Disease and Inflammation in Aging (PUFA Trial): Rationale, Design and Baseline Results. Nutrients. 2019;11(4):735

8. Bowman GL, Murchison C, Silbert L, et al. Effects of omega-3 (n-3) polyunsaturated fatty acids (PUFA) on cerebral white matter hyperintensities, medial temporal lobe atrophy and white matter integrity in older nondemented adults: A 3-year randomizedcontrolled phase 2 trial. J Prev $\mathrm{Alz}$ Dis 2020; 7(S1):S18.

9. Andrieu S, Guyonnet S, Coley N, et al. Effect of long-term omega 3 polyunsaturated fatty acid supplementation with or without multidomain 
intervention on cognitive function in elderly adults with memory complaints (MAPT): a randomised, placebo-controlled trial. Lancet Neurol. 2017;16(5):377-89.

10. Delrieu J, Payoux P, Carrié I, et al. Multidomain intervention and/or omega3 in nondemented elderly subjects according to amyloid status. Alzheimers Dement J Alzheimers Assoc. 2019;15(11):1392-401.

11. Nakamura A, Kaneko N, Villemagne VL, et al. High performance plasma amyloid- $\beta$ biomarkers for Alzheimer's disease. Nature. 2018;554(7691):249-54

12. Bateman RJ, Blennow K, Doody R, et al. Plasma Biomarkers of AD Emerging as Essential Tools for Drug Development: An EU/US CTAD Task Force Report. J Prev Alzheimers Dis. 2019;6(3):169-73.

13. Wang X, Sun Y, Li T, Cai Y, Han Y. Amyloid- $\beta$ as a Blood Biomarker for Alzheimer's Disease: A Review of Recent Literature. J Alzheimers Dis JAD. 2020;73(3):819-32.

14. Delrieu J, Vellas B, Cantet C, Bateman R, Andrieu S. Multidomain intervention and/or omega 3 in non-demented subjects according to plasma A $\beta 42 / 40$ ratio: cognitive impact at 3 and 5 years in a subgroup analysis from the randomized clinical MAPT trial. J Prev Alz Dis 2020; 7(S1):S48-S49.

15. Pan Y, Araujo JA, Burrows J, et al. Cognitive enhancement in middle-aged and old cats with dietary supplementation with a nutrient blend containing fish oil, B vitamins, antioxidants and arginine. Br J Nutr. 2013;110(1):40-9.

16. Pan Y, Kennedy AD, Jönsson TJ, Milgram NW. Cognitive enhancement in old dogs from dietary supplementation with a nutrient blend containing arginine, antioxidants, B vitamins and fish oil. Br J Nutr. 2018;119(3):349-58.

17. Smith AD, Smith SM, de Jager CA, et al. Homocysteine-lowering by B vitamins slows the rate of accelerated brain atrophy in mild cognitive impairment: a randomized controlled trial. PloS One. 2010;5(9):e12244.

18. Oulhaj A, Jernerén F, Refsum H, Smith AD, de Jager CA. Omega-3 Fatty Acid Status Enhances the Prevention of Cognitive Decline by B Vitamins in Mild Cognitive Impairment. J Alzheimers Dis JAD. 2016;50(2):547-57.

19. Li M, Li W, Gao Y, et al. Effect of folic acid combined with docosahexaenoic acid intervention on mild cognitive impairment in elderly: a randomized double-blind, placebo-controlled trial. Eur J Nutr. 2020 Aug 28; doi: 10.1007/ s00394-020-02373-3. Online ahead of print.

20. Soininen H, Solomon A, Visser PJ, et al. 24-month intervention with a specific multinutrient in people with prodromal Alzheimer's disease (LipiDiDiet): a randomised, double-blind, controlled trial. Lancet Neurol. 2017;16(12):965-75.

21. Hartmann T, Solomon A, Visser P, et al. LipiDiDiet results: 3-year evaluation of Fortasyn Connect in individuals with prodromal Alzheimer's Disease. 2020; J Prev Alz Dis 2020; 7(S1):S15-S16.

22. Soininen H, Solomon A, Visser PJ, et al. 36-month LipiDiDiet multinutrient clinical trial in prodromal Alzheimer's disease. Alzheimers Dement. 2021;17(1):29-40.

23. Smith AD, Refsum H. Homocysteine, B Vitamins, and Cognitive Impairment. Annu Rev Nutr. 2016;36:211-39.

24. Setién-Suero E, Suárez-Pinilla M, Suárez-Pinilla P, Crespo-Facorro B, AyesaArriola R. Homocysteine and cognition: A systematic review of 111 studies. Neurosci Biobehav Rev. 2016;69:280-98.

25. Hooper C, De Souto Barreto P, Pahor M, Weiner M, Vellas B. The Relationship of Omega 3 Polyunsaturated Fatty Acids in Red Blood Cell Membranes with Cognitive Function and Brain Structure: A Review Focussed on Alzheimer's Disease. J Prev Alzheimers Dis. 2018;5(1):78-84.

26. Giudici KV, Guyonnet S, Cantet C, et al. A 1-year randomized controlled trial of a nutritional blend to prevent cognitive decline among communitydwelling older adults: the NOLAN Study. J Prev Alz Dis 2020; 7(S1):S43-S44.
27. Owen OE, Morgan AP, Kemp HG, Sullivan JM, Herrera MG, Cahill GF. Brain metabolism during fasting. J Clin Invest. 1967;46(10):1589-95.

28. Castellano C-A, Nugent S, Paquet $\mathrm{N}$, et al. Lower brain 18F-fluorodeoxyglucose uptake but normal 11C-acetoacetate metabolism in mild Alzheimer's disease dementia. J Alzheimers Dis JAD. 2015;43(4):1343-53.

29. Cunnane SC, Courchesne-Loyer A, St-Pierre V, et al. Can ketones compensate for deteriorating brain glucose uptake during aging? Implications for the risk and treatment of Alzheimer's disease. Ann N Y Acad Sci. 2016;1367(1):12-20.

30. Croteau E, Castellano CA, Fortier M, et al. A cross-sectional comparison of brain glucose and ketone metabolism in cognitively healthy older adults, mild cognitive impairment and early Alzheimer's disease. Exp Gerontol. 2018;107:18-26.

31. Cunnane SC, Trushina E, Morland C, et al. Brain energy rescue: an emerging therapeutic concept for neurodegenerative disorders of ageing. Nat Rev Drug Discov. 2020;19(9):609-33.

32. Avgerinos KI, Egan JM, Mattson MP, Kapogiannis D. Medium Chain Triglycerides induce mild ketosis and may improve cognition in Alzheimer's disease. A systematic review and meta-analysis of human studies. Ageing Res Rev. 2020;58:101001.

33. Cunnane S, Fortier M, Castellano A, et al. Ketones improve brain energetics and cognitive performance in mild cognitive impairment: Final results of the 6-month Benefic trial in MCI. J Prev Alz Dis 2020; 7(S1):S10-S11.

34. Fortier M, Castellano C-A, St-Pierre V, et al. A ketogenic drink improves cognition in mild cognitive impairment: Results of a 6-month RCT. Alzheimers Dement. 2020 Oct 26; doi: 10.1002/alz.12206. Online ahead of print.

35. Xue $\mathrm{M}, \mathrm{Xu} \mathrm{W}, \mathrm{Ou} \mathrm{Y}-\mathrm{N}$, et al. Diabetes mellitus and risks of cognitive impairment and dementia: A systematic review and meta-analysis of 144 prospective studies. Ageing Res Rev. 2019;55:100944.

36. Cooper C, Sommerlad A, Lyketsos CG, Livingston G. Modifiable predictors of dementia in mild cognitive impairment: a systematic review and metaanalysis. Am J Psychiatry. 2015;172(4):323-34.

37. Pal K, Mukadam N, Petersen I, Cooper C. Mild cognitive impairment and progression to dementia in people with diabetes, prediabetes and metabolic syndrome: a systematic review and meta-analysis. Soc Psychiatry Psychiatr Epidemiol. 2018;53(11):1149-60.

38. Biessels GJ, Staekenborg S, Brunner E, Brayne C, Scheltens P. Risk of dementia in diabetes mellitus: a systematic review. Lancet Neurol. 2006;5(1):64-74.

39. Heston MB, Gaitan JM, Ma Y, et al. Exercise and carbohydrate-restricted diet associates with improved insulin resistance and cognitive performance. J Prev Alz Dis 2020; 7(S1):S89.

40. Fávaro-Moreira NC, Krausch-Hofmann S, Matthys C, et al. Risk Factors for Malnutrition in Older Adults: A Systematic Review of the Literature Based on Longitudinal Data123. Adv Nutr. 2016;7(3):507-22.

41. Borg S ter, Verlaan S, Hemsworth J, et al. Micronutrient intakes and potential inadequacies of community-dwelling older adults: a systematic review. Br J Nutr. 2015;113(8):1195-206.

42. Vural Z, Avery A, Kalogiros DI, Coneyworth LJ, Welham SJM. Trace Minera Intake and Deficiencies in Older Adults Living in the Community and Institutions: A Systematic Review. Nutrients. 2020;12(4):1072. 